\title{
Generation of sub-Poissonian photon number distribution
}

\section{Grønbech-Jensen, N.; Ramanujam, P. S.}

\section{Published in:}

Physical Review A

Link to article, DOI:

10.1103/PhysRevA.41.2906

Publication date:

1990

Document Version

Publisher's PDF, also known as Version of record

Link back to DTU Orbit

\section{Citation (APA):}

Grønbech-Jensen, N., \& Ramanujam, P. S. (1990). Generation of sub-Poissonian photon number distribution. Physical Review A, 41(5), 2906-2909. https://doi.org/10.1103/PhysRevA.41.2906

\section{General rights}

Copyright and moral rights for the publications made accessible in the public portal are retained by the authors and/or other copyright owners and it is a condition of accessing publications that users recognise and abide by the legal requirements associated with these rights.

- Users may download and print one copy of any publication from the public portal for the purpose of private study or research.

- You may not further distribute the material or use it for any profit-making activity or commercial gain

- You may freely distribute the URL identifying the publication in the public portal

If you believe that this document breaches copyright please contact us providing details, and we will remove access to the work immediately and investigate your claim 


\title{
Generation of sub-Poissonian photon number distribution
}

\author{
N. Grønbech-Jensen \\ Laboratory for Applied Mathematical Physics and Physics Laboratory I, The Technical University of Denmark, \\ 2800 Lyngby, Denmark \\ P. S. Ramanujam \\ Danish Institute of Fundamental Metrology, 2800 Lyngby, Denmark
}

(Received 4 December 1989)

\begin{abstract}
An optimization of a nonlinear Mach-Zehnder interferometer to produce sub-Poissonian photon number distribution is proposed. We treat the system quantum mechanically and estimate the mirror parameters, the nonlinearity of the medium in the interferometer, and the input power to obtain minimal output uncertainty in the photon number. The power efficiency of the system is shown to be high.
\end{abstract}

Squeezed states of light ${ }^{1}$ provide the possibility of studying states with small uncertainties in one observable, at the expense of a large uncertainty in the conjugate observable. Elliptical squeezed states (ESS), ${ }^{2}$ where the minimum product of the phase-quadrature uncertainties is given by $\left(\Delta \alpha_{1}\right)^{2}\left(\Delta \alpha_{2}\right)^{2}=\frac{1}{16}$, can exhibit sub-Poissonian statistics: $(\Delta n)^{2}<\langle\hat{n}\rangle$. It has been shown in Ref. 3 that photon number uncertainties less than $(\Delta n)^{2}=\langle\hat{n}\rangle^{2 / 3}$ cannot be achieved for these states.

However, Kitagawa and Yamamoto ${ }^{4}$ have demonstrated the possibility of obtaining a photon number uncertainty $(\Delta n)^{2} \sim\langle\hat{n}\rangle^{1 / 3}$ using a nonlinear photon-number-conserving interaction (Kerr interaction) followed by a coherent displacement in the phase space. This type of squeezed state, called crescent squeezed states (CSS), results in states that approach the number states directly, whereas the ESS approach one of the phase quadratures. Obviously there are some advantages in dealing with the CSS when a photon state with a low uncertainty is needed.

The system proposed in Ref. 4 is shown in Fig. 1. It consists of an interferometer, in one arm of which is placed a Kerr medium and in the other, a phase shifter. The mirrors $M_{1}$ and $M_{2}$ have a field transmittance and a reflectance $\left(\tau_{1}, \rho_{1}\right),\left(\tau_{2}, \rho_{2}\right)$, respectively, and $M_{3}$ and $M_{4}$ are $100 \%$ reflecting. The interaction Hamiltonian $\hat{H}_{i}$ of

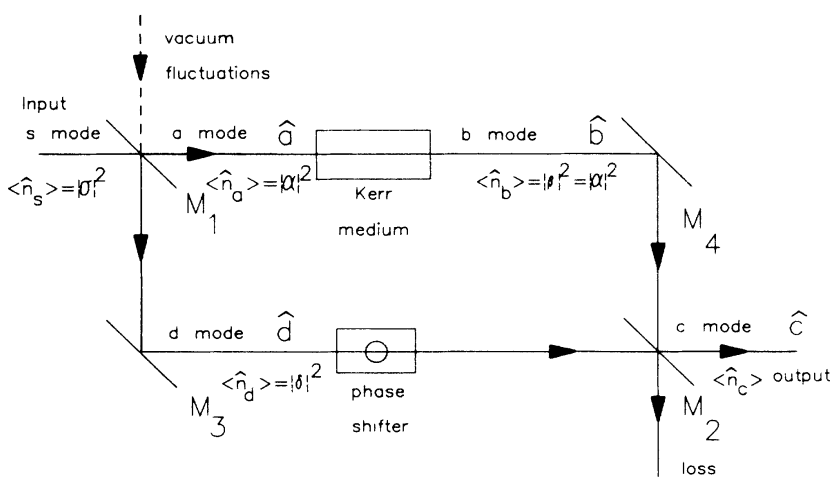

FIG. 1. Nonlinear Mach-Zehnder interferometer. the Kerr medium is given by

$$
\hat{H}_{i}=\hbar \chi_{\mathrm{NL}} \hat{a}^{\dagger 2} \hat{a}^{2},
$$

where $\hat{a}$ is the annihilation operator for the observed mode and $\chi_{\mathrm{NL}}$ is proportional to the third-order nonlinearity of the Kerr medium. ${ }^{5}$ This four-wave interaction, unlike a second-order interaction, is photon conserving and thus also conserves the photon number statistics. However, as shown, ${ }^{4}$ the mixing at $M_{2}$ gives rise to a collapse in the photon number uncertainty in the output $c$ mode.

In the Heisenberg picture, the annihilation operators for the different modes in the interferometer are given by assuming that the input mode $s$ is in a coherent state:

$$
|\sigma\rangle_{s}=\exp \left(-\frac{|\sigma|^{2}}{2}\right) \sum_{n=0}^{\infty} \frac{\sigma^{n}}{\sqrt{n !}}|n\rangle,
$$

where $\sigma$ is a complex number and $|n\rangle$ is the $n$th number state. Coherent states $|\alpha\rangle$ and $|\delta\rangle$ propagate as $a$ and $d$ modes giving rise to the energy conservation $|\alpha|^{2}$ $+|\delta|^{2}=|\sigma|^{2}$.

The $b$-mode state is given by the unitary transformation $U:$

$\hat{b}=U^{\dagger} \hat{a} U, U=\exp \left[\frac{1}{2} i \gamma \hat{n}(\hat{n}-1)\right], \hat{b}=\exp (i \hat{n} \gamma) \hat{a}$,

where $\hat{b}$ is the annihilation operator in the $b$ mode, and $\gamma$ is the normalized nonlinearity of the Kerr medium:

$$
\gamma=2 \frac{\chi_{\mathrm{NL}} L}{v} .
$$

Here $v$ is the propagation velocity and $L$ is the length of the Kerr medium.

If $\phi=|\alpha|^{2} \sin \gamma$ is the phase shift in the Kerr medium, ${ }^{6}$ and $\Delta$ is the phase difference between the $b$ mode and the $d$ mode at $M_{2}$, it was argued ${ }^{4}$ that the interaction at $M_{2}$ could, in the case of $|\delta|^{2} \rightarrow \infty$, be described by a displacement $\xi=\eta \alpha \exp [i(\phi+\Delta)]$, where $\eta$ is the mixing ratio, $|\delta| /|\beta|=|\delta| /|\alpha|$ in the $c$ mode. The optimal value of $\Delta$ was found to be $-\pi / 2$. Fixing the nonlinearity $\gamma$ and the photon number $|\alpha|^{2}$ in the Kerr medium, and assuming that $|\delta|^{2} \rightarrow \infty$, it was possible to minimize the 
Fano factor, $F=(\Delta n)^{2} /\langle\hat{n}\rangle$ (Ref. 7) with respect to $\eta$. The obtained minimal Fano factor was found to be $F_{\min } \sim\left\langle\hat{n}_{c}\right\rangle^{-2 / 3}$

The aim of the present paper is to demonstrate that even away from the limit $|\delta|^{2} \rightarrow \infty$, a considerable reduction in the photon number uncertainty can be found in the output state. This is done by fixing the photon number in the $s$ mode instead of in the $a$ mode.

We treat the mixing at $M_{2}$ as an interaction for the quantum-mechanical beam splitter, ${ }^{8}$ instead of as a simple displacement as is done in Ref. 4. Because of the orthogonality between the $b$ and the $d$ mode, $\hat{b}$ and $\hat{d}$ must commute. Choosing $\Delta=-\pi / 2$ by using the same arguments as in Ref. 4, we find the annihilation operator $\hat{c}$ in the $c$ mode as

$$
\begin{aligned}
& \hat{c}=\rho_{2} \hat{b}+\tau_{2} \hat{d} e^{i(\phi+\Delta)}, \\
& \hat{c}=\rho_{2} \exp \left(i \gamma \hat{n}_{a}\right) \hat{a}-i \tau_{2} \hat{d} \exp (i \phi) .
\end{aligned}
$$

The boson commutator relation $\left[\hat{c}^{\dagger}, \hat{c}\right]=1$ is fulfilled for the $c$ mode.

Using the following relations:

$$
\begin{aligned}
& \hat{a} \exp \left(i \gamma \hat{n}_{a}\right)=\exp (i \gamma) \exp \left(i \gamma \hat{n}_{a}\right) \hat{a}, \\
& \exp \left(i \gamma \hat{n}_{a}\right) \hat{a}^{\dagger}=\exp (i \gamma) \hat{a}^{\dagger} \exp \left(i \gamma \hat{n}_{a}\right), \\
& \left\langle\alpha\left|\exp \left(i \gamma \hat{n}_{a}\right)\right| \alpha\right\rangle=\exp \left(-|\alpha|^{2}\right) \exp \left[|\alpha|^{2} \exp (i \gamma)\right],
\end{aligned}
$$

we obtain

$$
\begin{aligned}
& \left\langle\hat{c}^{\dagger} \hat{c}\right\rangle=\left\langle\hat{n}_{c}\right\rangle-\rho_{2}^{2}|\alpha|^{2}+\tau_{2}^{2}|\delta|^{2}, \\
& \left\langle\hat{c}^{+2} \hat{c}^{2}\right\rangle=\rho_{2}^{4}|\alpha|^{4}-4 \tau_{2} \rho_{2}^{3}|\alpha|^{3}|\delta| \exp \left(-\frac{1}{2} r\right) \sin \gamma+2 \rho_{2}^{2} \tau_{2}^{2}|\alpha|^{2}|\delta|^{2}[2-\exp (-2 t) \cos (\gamma-r \sin \gamma)]+\tau_{2}^{4}|\delta|^{4}, \\
& r=4|\alpha|^{2} \sin ^{2}\left(\frac{1}{2} \gamma\right), t=|\alpha|^{2} \sin ^{2} \gamma .
\end{aligned}
$$

The following relations are obtained at the beam splitter $M_{1}$ :

$$
\begin{aligned}
& |\alpha|^{2}=\tau_{1}^{2}|\sigma|^{2}=|\beta|^{2}, \\
& |\delta|^{2}=\rho_{1}^{2}|\sigma|^{2}
\end{aligned}
$$

Note that a possible phase shift at $M_{1}$ is not important, because of the use of the phase shifter in the linear interferometer arm to adjust $\Delta=-\pi / 2$. Inserting Eq. (8) into Eq. (7), we find the Fano factor for the $c$ mode,

$$
F=\frac{(\Delta n)^{2}}{\left\langle\hat{n}_{c}\right\rangle}=\frac{\left\langle\hat{n}_{c}\right\rangle+\left\langle\hat{c}^{\dagger 2} \hat{c}^{2}\right\rangle-\left\langle\hat{n}_{c}\right\rangle^{2}}{\left\langle\hat{n}_{c}\right\rangle},
$$

which finally gives

$$
F=1-\frac{2 \tau_{1}^{3} \rho_{1} \tau_{2} \rho_{2}^{3} p-\tau_{1}^{2} \rho_{1}^{2} \tau_{2}^{2} \rho_{2}^{2} q}{\tau_{1}^{2} \rho_{2}^{2}+\rho_{1}^{2} \tau_{2}^{2}},
$$

where

$$
\begin{aligned}
& p=2|\sigma|^{2} \exp \left(-\frac{1}{2} r\right) \sin \gamma, \\
& q=2|\sigma|^{2}[1-\exp (-2 t) \cos (\gamma-r \sin \gamma)], \\
& r=4 \tau_{1}^{2}|\sigma|^{2} \sin ^{2}\left(\frac{1}{2} \gamma\right), \\
& t=\tau_{1}^{2}|\sigma|^{2} \sin ^{2} \gamma .
\end{aligned}
$$

The mean photon number in the $c$ mode $\left\langle\hat{n}_{c}\right\rangle$ and the ratio $\eta$ between the field contribution from the $b$ and the $d$ mode to the $c$ mode are given by

$$
\begin{aligned}
& \left\langle\hat{n}_{c}\right\rangle=|\sigma|^{2}\left(\tau_{1}^{2} \rho_{2}^{2}+\rho_{1}^{2} \tau_{2}^{2}\right), \\
& \eta=\frac{\rho_{1} \tau_{2}}{\tau_{1} \rho_{2}} .
\end{aligned}
$$

Fixing the input photon number $|\sigma|^{2}$ and the nonlinearity $\gamma$, we minimize the Fano factor $F$ [Eq. (9)] with respect to the independent variables $\rho_{1}$ and $\tau_{2}$.

The optimization of Eq. (9) is done numerically and the result is displayed in Fig. 2 where the minimized Fano factor and the optimized parameters $\rho_{1}, \tau_{2}$, and $\eta$ are shown as a function of $\gamma$ for four different values of $|\sigma|^{2}$.

It is clear that sub-Poissonian statistics are observed for the optimized parameters. We observe that $\rho_{1}$ and $\tau_{2}$ are small and decrease for increasing $|\sigma|^{2}$ in the $\gamma$ region of interest. The abrupt change in the mirror parameters above the optimal $\gamma$ values can be understood as a reaction of the system to the large nonlinearity. The system reacts by lowering the photon number in the Kerr medium (increasing $\rho_{1}$ ) and to compensate for the consequent higher photon number in the $d$ mode, the transmittance of mirror $M_{2}, \tau_{2}$ has to be decreased. Another important characteristic in Fig. 2 is that $\tau_{2}$ and $\rho_{1}$ are almost equal, for optimal values of $\gamma$. The minimized Fano factor and the optimized parameters $\tau_{2}, \rho_{1}$, and $\gamma$ as a function of $|\sigma|^{2}$ are displayed in Fig. 3. We observe that all the curves, for $|\sigma|^{2}$ values above $10^{3}$, are straight lines in the logarithmic plot. We find from Fig. 3(a) that

$$
\begin{aligned}
& F_{\min } \sim\left\langle\hat{n}_{s}\right\rangle^{-0.4}, \\
& \gamma_{\mathrm{opt}} \sim\left\langle\hat{n}_{s}\right\rangle^{-0.6} .
\end{aligned}
$$

Although this reduction is less than the one obtained by Kitagawa and Yamamoto, ${ }^{4}$ it still represents a smaller photon number uncertainty than the smallest attainable for elliptic squeezed states, $\left\langle\hat{n}_{c}\right\rangle^{-1 / 3}$. The most remarkable feature of our description is revealed when we calculate the power efficiency of the system, given that $\tau_{i}^{2}+\rho_{i}^{2}=1$,

$$
\begin{aligned}
& \frac{\left\langle\hat{n}_{c}\right\rangle}{|\sigma|^{2}}=\tau_{1}^{2} \rho_{2}^{2}+\rho_{1}^{2} \tau_{2}^{2}, \\
& \frac{\left\langle\hat{n}_{c}\right\rangle}{|\sigma|^{2}}=1+2 \rho_{1}^{2} \tau_{2}^{2}-\tau_{2}^{2}-\rho_{1}^{2} .
\end{aligned}
$$

From Fig. 3, we find $\tau_{2}^{2} \approx \rho_{1}^{2} \ll 1$ for reasonably large values of $|\sigma|^{2}$ and Eq. (13) reduces to

$$
\frac{\left\langle\hat{n}_{c}\right\rangle}{|\sigma|^{2}} \approx 1-2 \tau_{2}^{2} \approx 1 \text {. }
$$



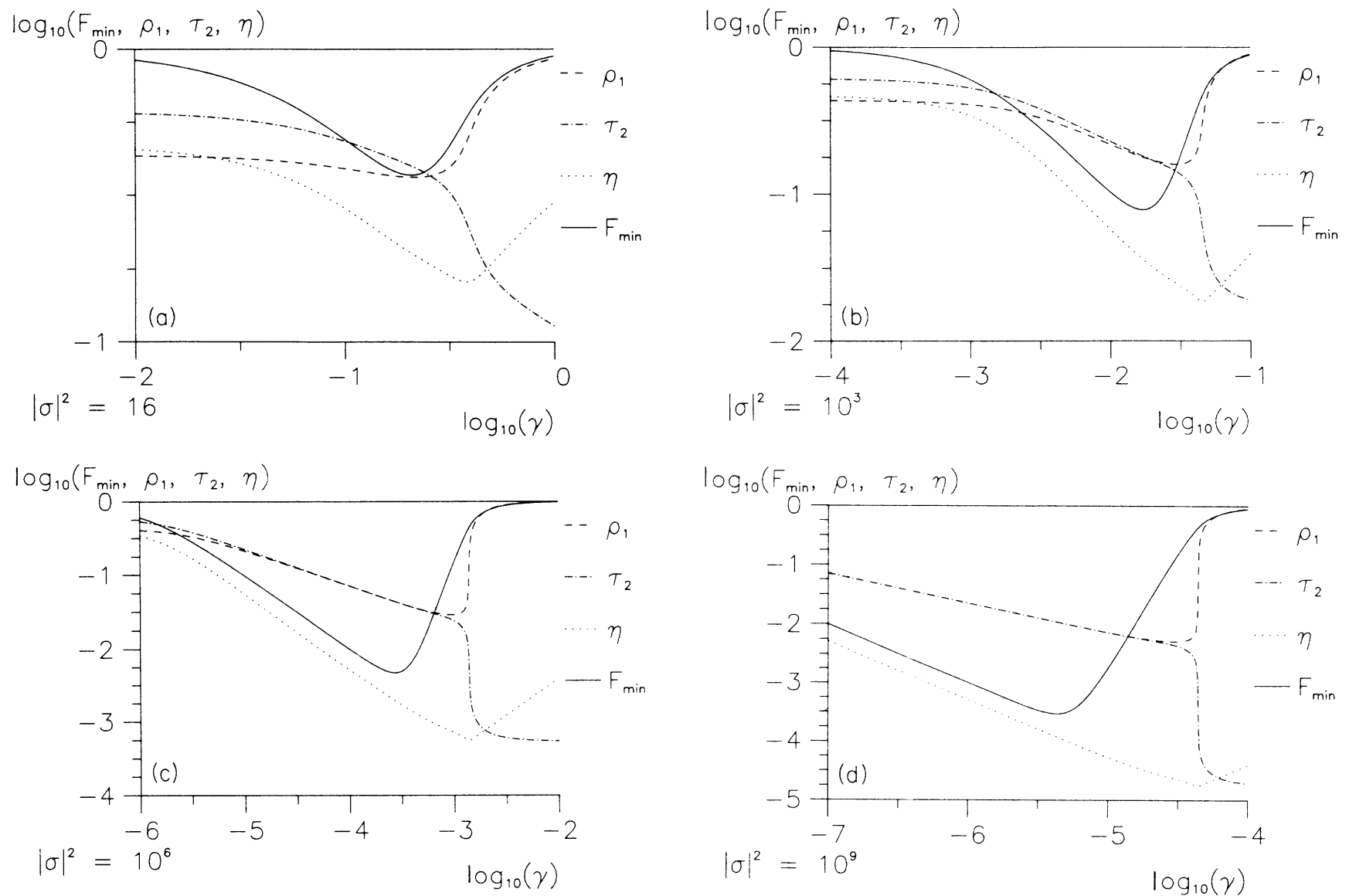

FIG. 2. Minimized Fano factor and optimized system parameters as a function of Kerr nonlinearity $\gamma$ for four input photon numbers: (a) $|\sigma|^{2}=16$, (b) $|\sigma|^{2}=10^{3}$, (c) $|\sigma|^{2}=10^{6}$, and (d) $|\sigma|^{2}=10^{9}$.

The output power in the $c$ mode can then be seen to be almost equal to the input power. This must be compared to the near-zero efficiency in Ref. 4.

We can make a rough estimate of the optimal mirror parameters by inserting $\tau_{2}^{2} \approx \rho_{1}^{2} \ll 1 \quad\left(\tau_{1} \approx 1\right)$ and $\gamma_{\mathrm{opt}}$ $=\left(|\sigma|^{2}\right)^{-0.6}$ into Eq. (9). The Fano factor becomes

$$
F \approx 1-2 \tau_{2}^{2} p+\tau_{2}^{4} q \text {. }
$$

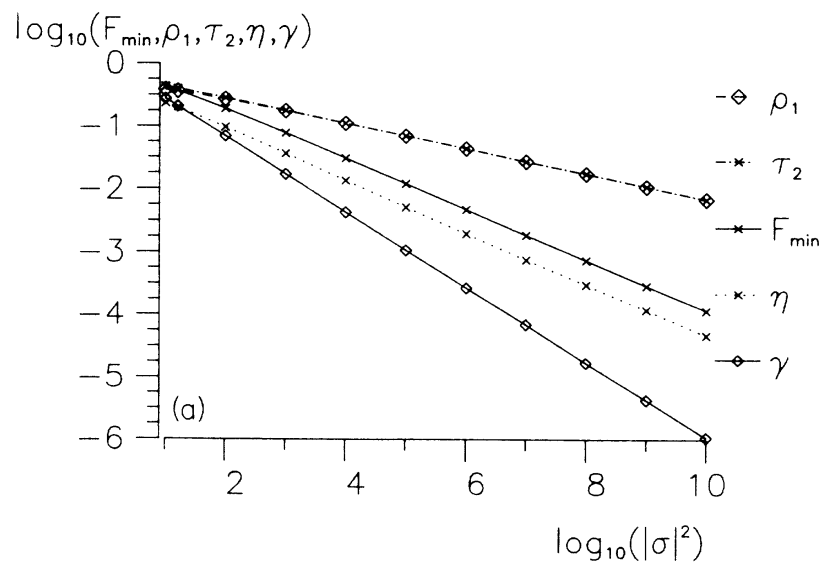

The minimum value of $F$ is obtained when

$$
\tau_{2}^{2} \approx p / q
$$

The above value is acceptable; however, if used in Eq. (15), a realistic behavior of $F$ is not found. This is due to the fact that the Fano factor is generated as a difference between two almost equal numbers. The approximations made in Eq. (9) will then give rather large relative varia-

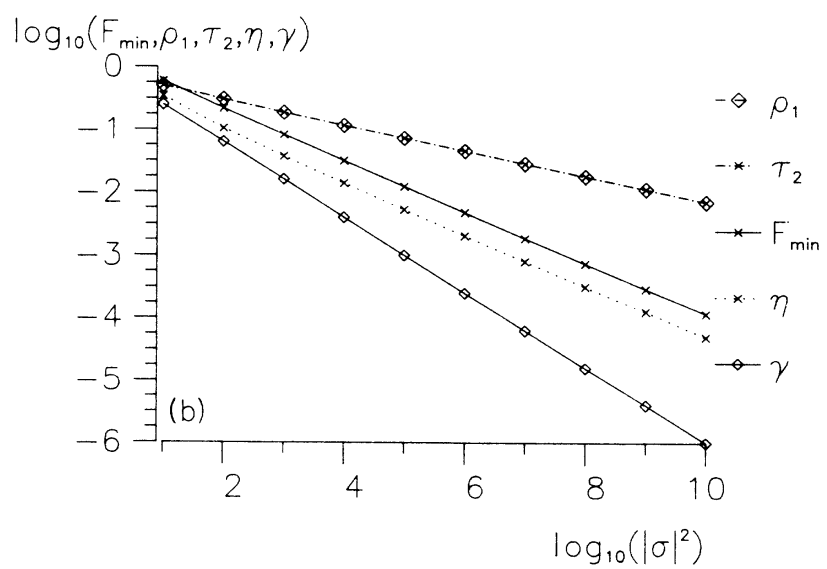

FIG. 3. Minimized Fano factor and optimized system parameters as a function of input photon number $|\sigma|^{2}$. (a) Numerical optimization, (b) "approximate" optimization. 
tions in $F$.

However, by using Eq. (16) and the optimal $\gamma$ value given by Eq. (12) in the true expression Eq. (9), we get Fig. 3(b) as an analog to Fig. 3(a). It is seen that the above analysis of the system gives the same values of $\tau_{2}$, $\rho_{1}, F_{\min }$, and $\eta$ for $|\sigma|^{2}$ above $10^{3}$. The plots differ in detail only for $|\sigma|^{2}$ below $10^{3}$. We have thus designed the system parameters for a given nonlinearity or given input/output mean photon number to get a sub-Poissonian output field. Given $\gamma\left(|\sigma|^{2}\right)$, one can use Eq. (12) to get $|\sigma|^{2}(\gamma)$. Then, using Eq. (16) and $\tau_{1}^{2} \approx 1$, the optimal parameters can be found. The system with $\Delta=-\pi / 2$ acts as a passive optical component that lowers the output field photon number uncertainty to $(\Delta n)^{2} \sim\langle\hat{n}\rangle^{0.6}$, with only a negligible power loss.

Finally, we shall derive the expression for the Fano factor in the case of infinite photon number in the $d$ mode as a special case of Eq. (9). To get $|\delta|^{2} \rightarrow \infty$, we must demand that $|\sigma|^{2} \rightarrow \infty$. The relevant fixed photon mean number is then $|\alpha|^{2}$. This implies that

$$
\begin{aligned}
& \tau_{1}=\frac{|\alpha|}{|\sigma|} \rightarrow 0, \\
& |\delta|^{2}=\left(1-\tau_{1}^{2}\right)|\sigma|^{2} \approx|\sigma|^{2} .
\end{aligned}
$$

We must now expect $\tau_{2}$ to be very small to prevent the large $d$-mode power getting transmitted into the $c$ mode. From Eq. (9) we find

$$
\begin{aligned}
& F \approx 1-\frac{2 \tilde{p} \tau_{2} / \tau_{1}-\tilde{q} \tau_{2}^{2} / \tau_{1}^{2}}{\left(1+\tau_{2}^{2} / \tau_{1}^{2}\right)}, \\
& F=1-\frac{2 \eta \tilde{p}-\tilde{q} \eta^{2}}{\left(1+\eta^{2}\right)},
\end{aligned}
$$

where the ratio of the field contribution $\eta$ is given by

$$
\eta=\frac{\rho_{1} \tau_{2}}{\tau_{1} \rho_{2}} \approx \frac{\tau_{2}}{\tau_{1}} ;\left(\tau_{1}, \tau_{2}\right) \ll 1,
$$

and the functions $\tilde{p}$ and $\tilde{q}$ are

$\tilde{p}=\tau_{1}^{2} p=2|\alpha|^{2} \exp \left(-\frac{1}{2} r\right) \sin \gamma$,

$\tilde{q}=\tau_{1}^{2} q=2|\alpha|^{2}[1-\exp (-2 t) \cos (\gamma-r \sin \gamma)]$.
Our Eq. (18) is identical to the expression for $F_{\min }$ discussed by Kitagawa and Yamamoto, ${ }^{4}$ where it was analytically optimized with respect to $\eta$ for fixed $|\alpha|^{2}$ :

$$
F_{\min }=1-\frac{\eta_{0}\left(2 \tilde{p}-\tilde{q} \eta_{0}\right)}{\left(1+\eta_{0}^{2}\right)}, \eta_{0}=\left[1+\left(\frac{\tilde{q}}{2 \tilde{p}}\right)^{2}\right]^{1 / 2}-\left(\frac{\tilde{q}}{2 \tilde{p}}\right) \text {. }
$$

This state, obtained by minimizing $F$ for $|\delta|^{2} \rightarrow \infty$, was shown in Ref. 4 to be a minimum uncertainty state with respect to phase and number, i.e., $\Delta \phi \Delta n \approx \frac{1}{4}$. This is not true for a state with a limited number of photons as in our case. An easy way to see this is to compare the optimal nonlinearity as a function of the number of photons in the Kerr medium in our treatment and the one in Ref. 4. For a given number, our optimization demands a slightly larger nonlinearity than Ref. 4. This results in a larger phase uncertainty of the $b$ mode ( $c$ mode). Moreover, we do not obtain a smaller uncertainty in the photon number to compensate for this increased phase uncertainty. Thus, the uncertainty product $\Delta \phi \Delta n$ is not minimized for the state obtained in this paper.

In conclusion, we have optimized the system proposed by Kitagawa and Yamamoto, ${ }^{4}$ with respect to the Kerr nonlinearity and the mirror parameters to obtain the minimum output photon number uncertainty, with a fixed input photon mean number in a coherent state. It is shown that the system produces an output state with Fano factor $F \approx\langle\hat{n}\rangle^{-0.4}$, which is better than the best obtainable using elliptical squeezed states. Moreover, the power lost in this process is found to be vanishingly small compared to the input power. Simple expressions for the optimal mirror parameters and the optimal nonlinearity are given. Finally, the expressions given in Ref. 4 for infinite input power are derived as a special case of our expressions.

We are pleased to acknowledge many interesting and instructive discussions with G. B. Jensen, Department of Electrophysics, The Technical University of Denmark, Lyngby, Denmark, and K. Mølmer, Department of Physics, University of Aarhus, Denmark. P. S. Ramanujam thanks the Danish Metrology Council for financial support.
${ }^{1}$ See, for example, special features issue, J. Opt. Soc. Am. B 4 (10) (1987).

${ }^{2}$ H. P. Yuen, Phys. Rev. A 13, 2226 (1976).

${ }^{3}$ R. S. Bondurant and J. H. Shapiro, Phys. Rev. D 30, 2548 (1984).

${ }^{4}$ M. Kitagawa and Y. Yamamoto, Phys. Rev. A 34, 3974 (1986).
${ }^{5}$ N. Imoto, H. A. Haus, and Y. Yamamoto, Phys. Rev. A 32, 2287 (1985).

${ }^{6}$ M. Kitagawa, N. Imoto, and Y. Yamamoto, Phys. Rev. A 35, 5270 (1987).

${ }^{7}$ M. C. Teich and B. E. A. Saleh, Opt. Lett. 7, 365 (1982).

${ }^{8}$ S. Prasad, M. O. Scully, and W. Martienssen, Opt. Commun. 62, 139 (1987). 